\title{
Transcriptome profiling reveals the candidate genes associated with aroma metabolites and emission of pear (Pyrus ussuriensis cv.)
}

Shuwei Wei ${ }^{1,2}$, Shutian Tao ${ }^{1}$, Gaihua Qin ${ }^{1}$, Shaomin Wang ${ }^{2}$, Jihan Tao ${ }^{2}$, Jun $\mathrm{Wu}^{1}$, Juyou $\mathrm{Wu}^{1}$, and Shaoling Zhang ${ }^{1 *}$

${ }^{1}$ College of Horticulture, State Key Laboratory of Crop Genetics and Germplasm Enhancement, Nanjing Agricultural University, Nanjing, China, 210095

${ }^{2}$ Shandong Institute of Pomology, Taian, China, 271000

Running head: Volatile aroma metabolites and emission-related gene identification in pear

Key words: pear, 'Nanguoli', aroma, metabolites, emission, digital gene expression (DGE), differentially expressed genes

*Corresponding author:

Shaoling Zhang:Tel.:+86-25-84396580; Fax:+86-25-84396485;

E-mail: slzhang@njau.edu.cn

Email addresses:

Shuwei Wei:weisw2007@163.com

Shutian Tao: shutian_tao@ hotmail.com

Gaihua Qin: qghahstu@163.com

Shaomin Wang: sdipwsm@163.com

Jihan Tao: sdtjh_69@163.com

Juyou Wu: juyouwu@njau.edu.cn

Jun Wu:wujun@njau.edu.cn 


\begin{abstract}
Aroma is an important factor affecting pear fruit quality. However, the molecular basis for the development of aroma metabolites and their emission is poorly understood. Unripe 'Nanguoli' pear fruit has poor aroma, while the ripe fruit has an intense aroma, and calcium treatment can enhance the aroma, providing good material for transcriptome analysis.

Digital gene expression (DGE) analysis of 'Nanguoli' fruit was performed at three important developmental stages, with and without calcium treatment, six libraries in total. Of the total number of mapped reads (11.4-13.6 million), an average of $69.81 \%$ could be mapped to the pear genome. A total of 10,776 differentially expressed genes between the poor aroma and intense aroma were categorized into 36 functional groups and 37 pathways according to GO and KEGG databases, respectively. Genes more highly expressed in the more aromatic fruit include some from the fatty acid pathway such as $F A D, A D H, P D C, L O X$, and lipase genes. Some transcription factors were identified with high correlation to aroma biosynthesis in pear. Also, ABA may play an important role in the calcium-triggered improvement of 'Nanguoli' aroma. In addition, candidate genes were verified with quantitative real-time PCR.
\end{abstract}

\title{
Introduction
}

Aroma is a decisive factor determining fruit quality and consumer acceptance (Zhang et al. 2007; Xi et al. 2012) and has gained increasing attention in recent years (Muna et al. 2013). 'Nanguoli', a variety of Pyrus ussuriensis-one of the four main cultivated species of pear in China-has a rich fragrance, but is very hard with little aroma when harvested at commercial harvest (unripe). Only after a post-harvest ripening process of about $8-10 \mathrm{~d}$ at $20^{\circ} \mathrm{C}$, the (ripe) fruit has intense aroma production, improving the 
final fruit quality.

The volatile aroma of pear has been studied since the report of Jennings et al. (1964), and many studies have investigated the characteristic aroma volatiles of different pear species or varieties (Qin et al. 2012), synthesis of aromatic compounds (Li et al. 2014) and also the effect of bagging and organic fertilizer (Wei et al. 2012), ripening stages (Kou et al. 2012) and storage (Zhang et al. 2013) on the aroma produced. The effects of calcium on fruit aroma have been reported: pre-harvest calcium spraying can improve volatile emission at commercial harvest of 'Fuji Kiku-8' apples (Ortiz et al. 2011); and emission of volatile esters of 'Golden Reinders' apple (Ortiz et al. 2010) after midterm storage can be improved by post-harvest calcium treatment. Our previous study also showed that spraying 'Nanguoli' fruit with $4 \%$ calcium chloride $15 \mathrm{~d}$ before harvest could promote the formation of aroma at harvest, in particular the formation of ester compounds that stimulate fruit flavor concentration.

Studies of enzyme activity, protein content, and expression of related genes indicated that production of aroma compounds was regulated by expression of key genes (Dudareva et al. 1998; Muna et al. 2013). Genes PuLOX1, PuLOX8, PuADH3, and PuAAT may also play important roles in ester formation of pear fruit ( $\mathrm{Li}$ et al. 2014). However, the metabolic process and emission of aromatic compounds is very complex. The key genes that control or regulate metabolites and emission of aroma compounds in pear are unknown, and an increased knowledge of gene expression and regulatory changes of 'Nanguoli' following post-harvest ripening and calcium treatment is necessary to understand the source of its strong aroma.

High-throughput sequencing is a revolutionary technological innovation, characterized by low cost and high data output, allowing practical genomics research (Elaine et al. 2008; Glenn et al. 2011). Digital gene expression (DGE) with high- 
throughput sequencing technologies is a novel and efficient approach to genome-wide expression profiling using next generation sequencing methodologies. This approach allows researchers to identify, quantify, and annotate expression at the whole genome level with or without prior sequence knowledge of any transcript from any organism, and has been used in many studies (Deng et al. 2014; Wang et al. 2014; Zhang et al. 2014). Overall, DGE dramatically enhances the identification of differentially expressed genes. Our laboratory previously completed whole genome sequencing (Wu et al. 2012) of pear 'Dangshansuli' (Pyrus bretschneideri Rehd.), which provided an invaluable new resource for biological research of Pyrus. In the present study, we established transcriptome datasets of poor and intense aroma pear fruit using DGE to gain a deeper insight into the molecular mechanism of pear fruit aroma biosynthesis and emission. We identified genes involved in fatty acid pathway, transcription factors, and other genes leading to improved aroma in 'Nanguoli' pear fruit. Our study presents a global survey of transcriptome profiles of aroma metabolites and emission in pear.

\section{Materials and methods}

\section{Plant materials and treatments}

For the characterization of aroma emission, 'Nanguoli' (Pyrus ussuriensis cv.) was selected for research. Fruits were provided by the National Germplasm Repository of Pear in the Research Institute of Pomology, Chinese Academy of Agricultural Sciences (CAAS), Xingcheng, Liaoning Province, China. On September 5, 2013 (15 days before harvest), six uniform ten-year-old 'Nanguoli' trees were selected and divided into two groups: one was treated with calcium, while the second one was maintained as control. Same size and color fruits from different sides of experimental trees were sprayed with calcium chloride $4 \%(\mathrm{~W} / \mathrm{V})$ on fruit surface, and the other 
three trees were sprayed with water as control (CK). Orchard management procedures, such as fertilization and irrigation, were the same for all treatments. Healthy fruits were harvested in commercial ripening period (135 days after pollination) and five days before harvest (130 days after pollination) by hand-picking, with all fruits picked from the southern or western crown, about $2 \mathrm{~m}$ above the ground. Individually packed fruit were taken to the laboratory immediately after harvest. Half of the fruits harvested in commercial ripening were cored and seeded and the skin and flesh were frozen with liquid nitrogen and stored in $-80^{\circ} \mathrm{C}$ freezer. The other half of the fruits harvested in commercial ripening were kept in $20^{\circ} \mathrm{C}$ for five days of post-harvest ripening, at the end of which they were cored and seeded, while the skin and flesh was frozen with liquid nitrogen and stored in a $-80^{\circ} \mathrm{C}$ freezer. For each treatment, 3-5 pears were combined for each of three replicates.

\section{Extraction and concentration of volatile aroma compounds}

GC-MS and HS-SPME were used for the extraction and concentration of volatile aroma compounds, following the methods reported by Qin (2012). SPME fibres coated with a $65 \mu \mathrm{m}$ thickness of polydimethylsiloxane-divinylbenzene $(65 \mu \mathrm{m}$ PDMS/DVB; Supelco Co., Bellefonte, PA, USA) were used in this study and activated before sampling according to the manufacturer's instructions. The core and seeds of each pear were removed and discarded; the skin and flesh was ground with a commercial blender to pulp for the extraction and concentration of volatile aromatic compounds. For each extraction, $10 \mathrm{~g}$ of pulp were placed into a $20 \mathrm{ml}$ screw-cap vial containing $3.6 \mathrm{~g}$ of $\mathrm{NaCl}$ to facilitate the release of volatiles compounds. Prior to sealing of the vials, $50 \mu \mathrm{l}$ of $0.04 \mathrm{~g} \mathrm{ml}^{-1} 3$-nonanone was added as internal standard, and mixed with a glass rod. A magnetic follower was added to each vial, which was placed into a constant-temperature water bath at $40^{\circ} \mathrm{C}$ with stirring. The SPME fibre 
was exposed to the head space of the sample for $30 \mathrm{~min}$ to adsorb the analyte, then introduced into the heated injector port of the chromatograph for desorption at $250{ }^{\circ} \mathrm{C}$ for $5 \mathrm{~min}$ in splitless mode.

\section{GC-MS conditions}

The volatile constituents were analyzed with an Agilent 5973B mass selective detector coupled to an Agilent 7890A gas chromatograph, equipped with a $30 \mathrm{~m} \times$ $0.25 \mathrm{~mm} \times 1.0 \mathrm{~mm}$ HP-5 MS (5\%phenyl-polymethylsiloxane) capillary column. A constant column flow of $1.0 \mathrm{ml} / \mathrm{min}$ helium was used as carrier gas. The injector and detector temperature were 250 and $280^{\circ} \mathrm{C}$, respectively. The oven temperature program was $35^{\circ} \mathrm{C}$ for $8 \mathrm{~min}$, increased at $2{ }^{\circ} \mathrm{C} / \mathrm{min}$ to $140{ }^{\circ} \mathrm{C}, 140{ }^{\circ} \mathrm{C}$ for $2 \mathrm{~min}$, then increased at $10{ }^{\circ} \mathrm{C} / \mathrm{min}$ to $260^{\circ} \mathrm{Cand}$ held for $5 \mathrm{~min}$. Mass spectra were recorded at 70 $\mathrm{eV}$ in electron impact (EI) ionization mode. The temperatures of quadrupole mass detector and ion source were 150 and $230{ }^{\circ} \mathrm{C}$, respectively. The temperature of transfer line was $280{ }^{\circ} \mathrm{C}$. Mass spectra were scanned in the $\mathrm{m} / \mathrm{z}$ range $33-350$ amu at intervals of $1 \mathrm{~s}$. Tentative identification of the volatile components was done by comparing the mass spectra of the samples with the data system library (NIST 98). Whenever it was possible, MS identification was confirmed with authentic references.

Quantification was done by the internal standard method, where the concentration of each volatile aromatic compound was normalized to that of 3-nonanone.

\section{DGE-tag profiling}

Total RNA was isolated from all the samples and libraries were named (table 1). DGE-tag profiling was carried out as described by Qi et al. (2013). Total RNA was extracted from the samples using Plant RNA Isolation Kit (AutoLab), followed by RNA purification with RNeasy MiniElute Cleanup Kit (Qiagen), according to the manufacturer's instructions. RNA degradation and contamination was monitored on 
$1 \%$ agarose gels. RNA purity was checked using the NanoPhotometer ${ }^{\circledR}$ spectrophotometer (IMPLEN, CA, USA). RNA concentration was measured using Qubit $^{\circledR}$ RNA Assay Kit in Qubit $^{\circledR} 2.0$ Flurometer (Life Technologies, CA, USA). RNA integrity was assessed using the RNA Nano 6000 Assay Kit of the Agilent Bioanalyzer 2100 system (Agilent Technologies, CA, USA).

\section{Library preparation for DGE sequencing}

A total amount of $3 \mu \mathrm{g}$ RNA per sample was used as input material for the RNA sample preparations. Sequencing libraries were generated using NEBNext ${ }^{\circledR}$ Ultra $^{\mathrm{TM}}$ RNA Library Prep Kit for Illumina ${ }^{\circledR}$ (NEB, USA) following manufacturer's recommendations and index codes were added to attribute sequences to each sample. Briefly, mRNA was purified from total RNA using poly-T oligo-attached magnetic beads. Fragmentation was carried out using divalent cations under elevated temperature in NEBNext First Strand Synthesis Reaction Buffer (5X). First strand cDNA was synthesized using random hexamer primer and M-MuLV Reverse Transcriptase $\left(\right.$ RNase $\left.\mathrm{H}^{-}\right)$. Second strand cDNA synthesis was subsequently performed using DNA Polymerase I and RNase H. The remaining overhangs were converted into blunt ends via exonuclease/polymerase. After adenylation of 3' ends of DNA fragments, NEBNext Adaptor for hairpin loop structure were ligated to prepare for hybridization. In order to select cDNA fragments of 150 200 bp in length, the library fragments were purified with AMPure XP system (Beckman Coulter, Beverly, USA). Then $3 \mu$ U USER Enzyme (NEB, USA) was used with size-selected, adaptorligated cDNA at $37{ }^{\circ} \mathrm{C}$ for $15 \mathrm{~min}$ followed by $5 \mathrm{~min}$ at $95^{\circ} \mathrm{C}$ before PCR. Then PCR was performed with Phusion High-Fidelity DNA polymerase, Universal PCR primers and Index (X) Primer. Lastly, PCR products were purified (AMPure XP system) and library quality was assessed on the Agilent Bioanalyzer 2100 system. Clustering of 
the index-coded samples was performed on a cBot Cluster Generation System using TruSeq SR Cluster Kit v3-cBot-HS (Illumina) according to the manufacturer's instructions. After cluster generation, the library preparations were sequenced on an Illumina Hiseq 2000/2500 platform and 100 bp/50bp single-end reads were generated.

\section{Data analysis}

Reference genome and gene model annotation files were downloaded from the genome website (http://202.195.250.6/center) directly. Index of the reference genome was built using Bowtie v2.0.6 and single-end clean reads were aligned to the reference genome using Top Hat v2.0.9, which can generate a database of splice junctions based on the gene model annotation file and thus a better mapping result than other nonsplice mapping tools. For unigene DGE, Bowtie v0.12.9 was used to align single-end clean reads to the unigene sequences. HTSeq v0.5.4p3 was used to count the read numbers mapped to each gene. The RPKM of each gene was then calculated based on the length of the gene and reads count mapped to this gene. To count the read number mapped to each unigene, RSEM (RNA-Seq by Expectation-Maximization) was used.

Differential expression analysis (for DESeq with biological replicates) of two conditions/groups (two biological replicates per condition) was performed using the DESeq $\mathrm{R}$ package (1.10.1). DESeq provides statistical routines for determining differential expression in DGE data using a model based on the negative binomial distribution. The resulting $P$-values were adjusted using Benjamini and Hochberg's approach for controlling the false discovery rate. Genes with an adjusted $P$-value $<0.05$ found by DESeq were assigned as differentially expressed.

For DEGSeq without biological replicates prior to differential gene expression analysis, the read counts were adjusted by edger program package through one scaling normalized factor for each sequenced library. 
Differential expression analysis of the two conditions was performed using the DEGSeq R package (1.12.0). The $P$-values were adjusted using the Benjamini \& Hochberg method. Corrected $P$-value of 0.005 and $\log 2$ (Fold change) of 1 were set as the threshold for significantly differential expression.

\section{GO and KEGG enrichment analysis of differentially expressed genes}

Gene Ontology (GO) enrichment analysis of differentially expressed genes was implemented by the GOseq R package, in which gene length bias was corrected. GO terms with corrected $P$-value less than 0.05 were considered significantly enriched by differentially expressed genes. We used KOBAS software to test the statistical enrichment of differentially expressed genes in Kyoto Encyclopedia of Genes and Genomes (KEGG) pathways (http://www.genome.jp/kegg/). PPI analysis of differentially expressed genes was based on the STRING database, which detects known and predicted Protein-Protein Interactions. For the species existing in the database, we constructed the networks by extracting the target gene list from the database. Blastx (v2.2.28) was used to align the target gene sequences to the selected reference protein sequences.

\section{qRT-PCR analysis}

To validate the expression patterns revealed by DEG results, 8 identified genes were analyzed using quantitative real-time PCR, qRT-PCR analysis was conducted using the Lightcycle-480 (Roche). The qPCR was performed on 3 biological sample replicates. The PCR reaction conditions were as follows: initiation with a $10 \mathrm{~min}$ denaturation at $95^{\circ} \mathrm{C}$ followed by 40 cycles of amplification with $15 \mathrm{~s}$ of denaturation at $95^{\circ} \mathrm{C}, 15 \mathrm{~s}$ of annealing at $60^{\circ} \mathrm{C}, 20 \mathrm{~s}$ of extension at $72^{\circ} \mathrm{C}$ and reading the plate for fluorescence data collection at $60^{\circ} \mathrm{C}$. A melting curve was performed from 60 to $95^{\circ} \mathrm{C}$ to check the specificity to the amplified product. All reactions were performed using 
the SYBRGREEN MasterMix (SYBR Premix EX Taq ${ }^{\mathrm{TM}}$, TaKaRa). Primers used for amplifying each structural gene and transcript factor are presented in Additional file 5.

qRT-PCR amplification and analysis were performed using IQ2 (Bio-Rad, USA). According to the manufacturer's instructions, fluorescence was measured at the end of each annealing step. Amplification was followed by melting curve analysis with continual fluorescence data acquisition during the $56-61{ }^{\circ} \mathrm{C}$ melt. The raw data were analyzed with iQ5 software (version 2.0, Bio-Rad), and the gene expression levels were normalized to Pyrus tubulin (accession number AY338250) to minimize variation in the cDNA template. qPCR data are technical replicates $(n=3)$ with error bars, representing means \pm SE (Additional file 4). Statistical and correlation analyses were performed with SPSS for Windows NT (release 8.0.0).

\section{Results}

\section{Effects of calcium treatments on fruit quality of 'Nanguoli'}

Differences in fruit quality between the calcium treatments and control (Table 2) included improved firmness, soluble solids and titratable acids of 'Nanguoli' fruit with calcium treatment. The soluble solids content of 'Nanguoli' fruit F5L_Ca was $12.53 \%, 6.28 \%$ greater than controls F5L_CK. We also found that the firmness was 38.16\% higher in F5L_Ca compared to F5L_CK.

\section{Effects of calcium treatments on aroma emission and ethylene production}

There were significant differences in aroma emission between the calcium treatments and control (Table 3; Fig. 1A). Post-harvest ripening remarkably increased the volatile aroma emission, and calcium treatment also significantly promoted aroma emission of 'Nanguoli' fruit. The total content of volatile aroma compounds (including esters, alcohols, aldehydes and ketones) of 'Nanguoli' fruit F5L_Ca was $6676.80 \mathrm{ng} / \mathrm{g}$, or $24.16 \%$ greater than controls F5L_CK and $68.61 \%$ more than F5_Ca. 
We also found that the acetate esters were particularly favored in pear fruit for instance (Fig. 1C), being 31.62\% higher in F5L_Ca compared to F5L_CK.

Ethylene $\left(\mathrm{C}_{2} \mathrm{H}_{4}\right)$ played an important role in regulating aroma metabolites and emission. The pre-harvest calcium treatment improved the aroma emission of 'Nanguoli' fruit, but suppressed its $\mathrm{C}_{2} \mathrm{H}_{4}$ production (Fig. 1B). Ethylene production of 'Nanguoli' fruit F5L_Ca was $105.0 \mu \mathrm{l} / \mathrm{kg} \cdot \mathrm{h}$, which was $22.22 \%$ less than F5L_CK $(135.0 \mu \mathrm{l} / \mathrm{kg} \cdot \mathrm{h})$.

\section{DGE library sequencing}

To obtain a global view of fruit transcriptomic response to calcium treatment, Illumina DGE analysis was performed for calcium-treated and control fruit at three ripening stages (Table 1). Fruit digital transcript abundance measurement libraries (Table 4) were deep sequenced and the six DGE libraries generated approximately 1619 million clean reads per library after filtering of the raw reads. The total number of mapped reads in each library in the range of 11.4-13.6 million, and an average of $69.81 \%$ (Table 5) of all clean data for the six libraries could be mapped to the pear reference genome (Wu et al. 2012). Among them, the number of unique matched reads was 9.0-11.8 million (Table 4). The Q30 percentages (a Q-score of 30\% corresponds to an error rate of 0.001 ) of all six libraries were $91.08-91.77 \%$ and the error rate was $<0.04 \%$. GC content was $46.44-47.93 \%$.

\section{Analysis of gene expression differential}

To compare differential expression patterns between the six libraries, we employed the DEGSeq R package (1.12.0). Prior to DGE analysis, for each sequenced library, the read counts were adjusted using the Edge R-program package through one scaling normalized factor.

We compared the six libraries: two treatments during the same stage, and the same 
treatment during different stages, so that seven pairs of comparisons were implemented. Among these seven comparisons, 38-2759 genes showed significant changes in expression, with an average of 1539 (Fig. 2). The differential expression pattern among libraries revealed that the largest differences were between F5_Ca and F5L_Ca, where 2759 genes were significantly differentially expressed. Of these genes, 1746 were up-regulated and 1013 down-regulated. The smallest difference was observed between F5L_Ca and F5L_CK, in which only 38 differentially expressed genes were identified, including 13 up-regulated and 25 down-regulated. We also observed specifically expressed genes (SEGs) between each pair of libraries (Fig. 3). Comparisons showed that there were 30-776 SEGs with an average of 373 among the three comparisons. The most SEGs were 776 for the comparison of F5B_CaVs F5_Ca is 776, and the least was 30 for the comparison of F5L_CaVsF5L_CK.

\section{Functional annotation of differentially expressed genes}

GO is an international standardized gene function classification system that describes properties of genes and their products in any organism. In this study GO analysis categorized a total of 12,311 differentially expressed genes into 36 functional groups (Fig. 4). The major subcategories were as follows: 10 subcategories for 'molecular function', nine for 'cellular components' and 17 for 'biological process'.

These results indicate that expressed genes functioning in 'biological process', 'metabolic process', 'biological regulation' and 'cellular process' were important during the aroma emission process (Additional file 2).

To further investigate the function of differentially expressed genes during aroma emission, significantly enriched KEGG pathways were identified according to $P$ values and enrichment factors. BLAST analysis against the KEGG database indicated that the expressed genes were involved in 37 pathways (Additional file 3). These 
genes were related to secondary metabolism, such as flavonoid biosynthesis; butanoate metabolism; carotenoid biosynthesis; glycine, serine and threonine metabolism; flavone and flavonol biosynthesis; brassinosteroid (BR) biosynthesis; plant circadian rhythm; C5-branched dibasic acid metabolism; and thiamine metabolism.

In addition, several biological processes not previously reported as associated with aroma emission, such as BR biosynthesis, plant circadian rhythm and steroid biosynthesis were found dramatically changed during aroma emission response to calcium treatment or the post-harvest ripening process. These might be novel genes relevant to the aroma emission process in 'Nanguoli' fruit.

\section{Genes involved in the fatty acid pathway}

Many genes involved in fatty acid pathway were differentially expressed in response to post-harvest ripening or calcium treatment. Including three genes encoding fatty acid desaturase (omega-3 FAD, Pbr033732.1; omega-6 FAD, Pbr035830.1; and delta(8)-fatty-acid desaturase, Pbr005591.1), two genes encoding lipase(Pbr020255.1, lipase member N; and Pbr005807.1, esterase/lipase), Three genes encoding lipoxygenase (Pbr004967.1, Pbr005350.1 and Pbr008100.1), five genes related to acyl-CoA (Pbr016062.1, br006825.1, Pbr017584.1, Pbr013555.1 and Pbr003609.1) two genes encoding pyruvate decarboxylase (Pbr016162.1 and Pbr019445.1), one gene (Pbr030638.1) encoding allene oxide cyclase, two genes encoding fatty acylCoA reductase and 3-ketoacyl-CoA synthase (Pbr003500.1 and Pbr028833.1) were up-regulated by calcium treatment, and six genes related to ADH (Pbr039378.1, Pbr027959.1, Pbr039379.1, Pbr032983.1, Pbr034871.1 and Pbr027745.1) were differentially expressed, including up-regulated or down-regulated by calcium treatment. 


\section{Genes involved in the amino acid pathway}

In the present study, 28 genes related to amino acid metabolism (e.g. alanineglyoxylate aminotransferase, L-3-cyanoalanine synthase, leucine-rich repeat extensinlike protein 4 and phenylalanine ammonia-lyase 1) were differentially expressed, including 26 genes up-regulated and 2 genes down-regulated by calcium treatment.

\section{Genes involved in glycometabolism}

In the present study, DGE analysis showed that 25 genes related to glycometabolism were differentially expressed between calcium treatments and controls (Additional file 1), including those for fructokinase, lactonase, isomerase and dehydrogenase.

\section{Genes associated with photosynthesis}

Our DGE results showed that 168 genes encoding photosynthesis-related genes were differentially expressed under different treatments (Additional file 3), including 19 genes encoding photosynthesis-related genes that were up-regulated by calcium treatment (Additional file 1). Differentially expressed genes were found to be involved in the Photosystem I reaction center subunit, the Photosystem II reaction center, chlorophyll a-b binding protein and thylakoid membrane phosphoprotein (Additional file 1). We identified that calcium treatment led to strong induction in photosynthesis-related gene expression (Additional file 1).

\section{Genes associated with plant hormones}

We found that four genes associated with ABA (abscisic acid receptor PYL4 and 9, abscisic acid 8 and abscisic acid-insensitive 5) were differentially expressed, and all up-regulated by calcium treatment. Thus, ABA may play roles in the process of 'Nanguoli' aroma metabolites and emission.

Our research indicated that two genes (Pbr021939.1 and Pbr029668.1), encoding brassinosteroid LRR receptor kinase and brassinosteroid-regulated protein BRU1 
were up-regulated by calcium treatment at harvest, and a gene (Pbr030508.1) encoding brassinosteroid insensitive 1-associated receptor kinase 1 was up-regulated by calcium treatment at post-harvest. The expression of gene involved in cytochrome P450 90A1 (Pbr010897.1) increased by 2.41 times in calcium treatment compared to controls in 5 days before harvest.

\section{Genes encoding transcription factors}

In many biological processes, the expression of inducible genes is regulated at the transcriptional level by specific transcription factors. Our DGE results showed that 52 genes encoding transcription factors were differentially expressed (Additional file 1). These genes included ethylene-responsive factors ( $E R F s), b H L H$, Trihelix, $A S G, M Y B$, WRKY, UNE, HY, GATA, LHW, GTE, DIVARICATA, TCP, VOZ, E2FA, RAVI (AP2/ERF and B3 domain- containing) GLK, and MADS-box transcription factors.

The expression of three genes (Pbr022012.1, Pbr007181.1, and Pbr018801.2) encoding MADS-box transcription factor 3, agamous-like MADS-box protein AGL6 and agamous-like MADS-box protein AGL9 homolog, respectively, were specifically up-regulated during aroma emission. Twelve genes involved in ERFs (Pbr000396.1, Pbr001362.1, Pbr015699.1, Pbr019494.1, Pbr019669.1, Pbr024123.1, Pbr028836.1, Pbr030208.1, Pbr030451.1, Pbr033937.1, Pbr020024.1, and Pbr007473.1) were upregulated by calcium treatment. GLK2 (Pbr022508.1) was also up-regulated at harvest compared to $5 \mathrm{~d}$ post-harvest.

\section{Other genes associated with aroma metabolites and emission}

In the present study, we found one beta-glucosidase gene (beta-glucosidase 44, Pbr020361.1) and one gene (Pbr022329.1) encoding probable inactive betaglucosidase 14 significantly up-regulated at $5 \mathrm{~d}$ before harvest by the pre-harvest calcium treatment. One gene (Pbr007219.1) involved in the flavonoid biosynthesis 
pathway that was significantly up-regulated by calcium treatment. One gene (Pbr014973.1) related to calmodulin-binding transcription activator 2 was upregulated by calcium treatment, and we also found three $C D P K$ (calcium-dependent protein kinase) genes (Pbr021635.1, Pbr023342.1 and Pbr036114.1) and one gene (Pbr034360.1) encoding calmodulin-binding receptor-like cytoplasmic kinase 3 were up-regulated by calcium treatment-they may play a role in calcium improving aroma emission.

\section{Tag-mapped genes were confirmed by qRT-PCR}

To confirm the tag-mapped genes in the aroma metabolites and emission progress, 8 identified genes were analyzed using quantitative real-time PCR (Additional file 4). Representative genes selected for the analysis were those involved in beta-glucosidase, LOX, ADH, PDC,ABA, MADS-box and FAD. The results showed that although the exact fold-changes of eight of the selected genes at several data points varied between digital transcript abundance measurements and qRT-PCR analysis, the trends of gene expression detected by the two different approaches were largely consistent. The qRT-PCR further demonstrated that the candidate genes related to LOX (Pbr008100.1 and Pbr004967.1), ADH (Pbr039379.1), PDC (Pbr016162.1), beta-glucosidase

(Pbr020361.1), $\quad$ ABA(Pbr019415.1), $\quad$ FAD(Pbr033732.1), and MADS-box (Pbr007181.1)transcription factor genes showed significant differences between calcium treatments and participated in the process of metabolites and emission.

\section{Discussion}

The enzyme alcohol dehydrogenase $(\mathrm{ADH})$ has been shown to be responsible for the inter-conversion of aldehyde and alcohol forms of flavor volatiles (Chen et al. 1993; Speirs et al. 1998). ADHs in plants are encoded by a small gene family generally comprising two or three members (Thompson et al. 2007), and $A D H$ genes play a 
major role in flavor development in apple (Fabrizio et al. 2013), tomato (Speirs et al. 2002), grape (Tesniere et al. 2004), and melon fruit (Manriquez et al. 2006). Expression patterns of $A D H$ s differ during fruit development and ripening. MiADHI and $\mathrm{MiADH} 2$ transcripts accumulated at the onset of ripening in mango fruit, whereas MiADH3 accumulated during early development of fruit (Rajesh et al. 2010). In the present study, six genes related to ADH (Pbr039378.1, Pbr027959.1, Pbr039379.1, Pbr032983.1, Pbr034871.1 and Pbr027745.1) were differentially expressed, including being up- or down-regulated by calcium treatment, suggesting that ADH may play an important role in the process of aroma metabolites and emission.

In previous studies, PDC has been considered to be the rate-limiting factor in ethanol production, and has been suggested as an important enzyme in the switch to fermentative metabolism (Hossain et al. 1994). A significant increase in PDC activity during fruit ripening was correlated with increased ethanol production in 'Packham's Triumph' pears (Chervin et al. 1999). In the present study, two genes (Pbr016162.1 and Pbr019445.1) encoding pyruvate decarboxylase isozyme 1 (PDC1) were differentially expressed, and both PDC genes were significantly up-regulated by calcium treatment, suggesting that PDCs may play an important role in the process of aroma metabolism and emission.

Fatty acids are major precursors of volatile aroma in most fruit (Sanz et al. 1997). Fatty acids liberated by lipase activity and those further metabolized by $\beta$-oxidative enzymes and/or lipoxygenase (LOX) are generally regarded as the initial precursors of straight-chain esters, alcohols and aldehydes produced in fruit (Song et al. 2003). Ramarethinam et al. (2002) found that exogenous addition of lipase resulted in an increase in flavor volatiles of tea; and Marina et al. (2012) also found that recombinant Rhizopus oryzae lipase (rROL) catalyzed the synthesis of ethyl butyrate. 
In the present study, we found that two genes encoding lipase (Pbr020255.1, lipase member N; and Pbr005807.1, esterase/lipase) were up-regulated by calcium treatment.

The quantity of volatile aroma (Qin et al. 2014) was largely dependent on the quantity of precursors (linoleic and linolenic acids) present. Lee et al. (2012) reported that $\omega-6$ and $\omega-3$ fatty acid desaturase (FAD) played a key role in the synthesis of the unsaturated fatty acids linoleic (18:2) and linolenic (18:3) acids. Expression of the yeast $\Delta-9$ desaturase gene (Wang et al. 1996) led to changes in the levels of fatty acids (saturated and unsaturated) in tomato fruit (Lycopersicon esculentum Mill.), and changes in the profile of fatty acids were associated with flavor compounds derived from fatty acids, most notably cis-3-hexenol, 1-hexanol, hexanal and cis-3-hexenal. In the present study, we also found three genes encoding FAD (omega-3 FAD, Pbr033732.1; omega-6 FAD, Pbr035830.1; and delta(8)-fatty-acid desaturase, Pbr005591.1) were differentially expressed by calcium treatment. These results indicate that FAD may play an important role in the processing of pear aroma metabolites.

Previous studies have reported that the core reactions of the LOX pathway consist of the sequential action of lipase, LOX, hydroperoxide lyase (HPL) and alcohol dehydrogenase (ADH) (Matsui et al. 2000). Decreased LOX activity possibly leads to a shortage of lipid precursors for pear fruit ester biosynthesis (Lara et al. 2003). LOX catalyzes the bioxygenation of polyunsaturated fatty acids containing a cis,cis-1,4pentadiene unit to form conjugated hydroperoxydienoic acids. The LOX biosynthetic pathway has the potential to provide substrates for ester production. In tomato, TomloxC is a chloroplast-targeted LOX isoform that can use both linoleic and linolenic acids as substrates to generate volatile C6 flavor compounds, and is important in generation of fruit flavor (Chen et al. 2004). Li et al. (2014) reported that 
PuLOX1 and PuLOX8 may be critical for ester formation in pear. In the present study, we also found three genes (Pbr004967.1, Pbr005350.1, and Pbr008100.1) encoding LOX that were up-regulated by calcium treatment.

In many biological processes, the expression of inducible genes is regulated at the transcriptional level by specific transcription factors (TFs). Our DGE results showed that 52 genes encoding TFs were differentially expressed (Additional file 1). Qin et al. (2012) reported that MADS-box transcription factor RIN regulates fruit ripening by targeting specific molecular pathways, and modulates aroma formation by direct regulation of gene expression in the LOX pathway. Five genes (E8, TomloxC, PNAE, $P G K$, and $A D H 2$ ) were identified as direct targets of MADS-box transcription factor $R I N$, directly binding to the promoters of these genes. Tomlox $C$ and $A D H 2$, which encode LOX and $\mathrm{ADH}$, respectively, are critical for the production of characteristic tomato aromas derived from the LOX pathway. RIN also directly regulates the expression of $H P L$, another rate-limiting enzyme in the LOX pathway. In the present study, the expression of three genes (Pbr022012.1, Pbr007181.1, and Pbr018801.2) encoding MADS-box transcription factors were specifically up-regulated during aroma emission. Taken together, our findings suggest that MADS-box transcription factor 3, protein AGL9 and AGL6 may be new transcription factors regulating aroma emission.

Ethylene is central to ripening, inducing significant changes in gene expression (Michael et al. 1990). A genomics approach revealed that aroma production in apple is predominantly controlled by ethylene at the final step in each biosynthetic pathway (Robert et al. 2007). It has also been reported that pre-harvest spray treatments of $\mathrm{CaCl}_{2}$ inhibited ethylene production rates of 'Conference' pears at harvest, and after storage (Wójcik et al. 2014). In the present study, we also found that pre-harvest 
calcium treatment suppressed ethylene production in 'Nanguoli' fruit (Fig. 1B) at harvest and five days postharvest, which suggests that either 'Nanguoli' does not respond to ethylene, or that changes in volatiles are not a major effect. Li et al. (2014) found that the aroma production of 'Nanguoli' responds to ethylene, so we conclude that ethylene changes are not a major effect in 'Nanguoli' aroma emission.

Barickman et al. (2014) demonstrated that exogenous ABA treatments had a detrimental effect on aroma volatile concentrations. Expression analysis indicated that ABA treatment induced the MiADH1 gene of mango (Rajesh et al. 2010) by the ABA responsive ABRE motif (Gómez et al. 2010). Abscisic acid-insensitive 5 (ABI5) is involved in ABA signaling during seed maturation and germination (Sandra et al. 2005). We found that four genes associated with ABA were all up-regulated by calcium treatment. Thus, ABA may play a role in the process of 'Nanguoli' aroma metabolism and emission.

\section{Conclusions}

In this study, the transcriptome profile of $P$. ussuriensis 'Nanguoli' was investigated using Solexa/Illumina DGE deep sequencing. A list of candidate genes for functional studies involved in aroma biosynthesis, regulations and emission was generated, among which $A D H, P D C, L O X, F A D$, lipase, beta-glucosidase, and several TFs appeared to play a key role in promoting the aroma biosynthesis and emission pathway, and differentially expressed unigenes in different libraries were verified using qRT-PCR. ABA may play an important role in the process of calcium improvement of 'Nanguoli' aroma emission. Further studies should concentrate on functional characterization of these genes. This study offered new insights into the molecular mechanisms underlying pear aroma biosynthesis and emission. 


\section{Competing interests}

The authors declare that they have no competing interests.

\section{Authors' contributions}

SWW contributed to the experimental design and management, quantitative RT-PCR, data analysis and manuscript preparation. STT conducted the data analysis and organized and reviewed the manuscript. GHQ contributed to data analysis. SMW, JHT, JW and JYW prepared the plant materials and tissue collection. SLZ designed and managed the experiments. All authors have read and approved the final manuscript.

\section{Acknowledgements}

The work was financially supported by the National Science Foundation of China (31230063 , 31471839 and 31301739), National '863 project' (2013AA102606-02), the Earmarked Fund for China Agriculture Research System (CARS-29), the Youth Foundation of Shandong Academy of Agricultural Sciences (2015YQN40), science and technology development plan of Tai'an city (201440774).

\section{Author details}

${ }^{1}$ College of Horticulture, Nanjing Agricultural University, Nanjing, Jiangsu, People's Republic of China

${ }^{2}$ Institute of Shandong Pomology, Tai an, Shandong, People's Republic of China

\section{Data archiving statement}

Gene sequences of pear were obtained from the Pear Genome Project website (http://peargenome.njau.edu.cn/). Raw sequencing reads were deposited in the Sequence Read Archive (SRA) database in NCBI and accession numbers will be added when released. 


\section{References}

A.R.S. Chen, T. Chase,1993. Alcohol dehydrogenase 2 and pyruvate decarboxylase induction in ripening and hypoxic tomato fruit. J. Plant Physiol. Biochem. 31,875-885.

Barickman, Thomas Casey, 2014. The effect of abscisic acid on tomato calcium partitioning and fruit quality. $\mathrm{PhD}$ diss., University of Tennessee, http://trace.tennessee.edu/utk _graddiss/2675.

Bood K G., Zabetakis I., 2002. The biosynthesis of strawberry flavor (II): biosynthetic and molecular biology studies. J. Food Sci. 67, 2-8.

Chen GP, Rachel Hackett, David Walker, Andy Taylor, Lin ZF, and Donald Grierson. Identification of a specific isoform of tomato lipoxygenase (TomloxC) involved in the generation of fatty acid-derived flavor compounds. J. Plant Physiol. 2004,136, 26412651.

Chervin C, Truett JK, Speirs J. Alcohol dehydrogenase expression and alcohol production during pear ripening. J. Am. Soc. Hortic. Sci. 1999,124, 71-75.

Wang CL, Chin CK, Chi-Tang Ho, Chin-Fa Hwang, James J. Polashock,Charles E. Martin. Changes of fatty acids and fatty acid-derived flavor compounds by expressing the yeast $\Delta-9$ desaturase gene in tomato. J. Agric. Food. Chem. 1996,44, 3399-3402.

Carbone, F., Mourgues, F., Biasioli, F., Gasperi, F., Märk, T.D., Rosati, C., Perrotta, G.,. Development of molecular and biochemical tools to investigate fruit quality traits in strawberry elite genotypes. Molecular Breeding,2006,18:127-142.

Deng XX, Pan LQ ,Miao JJ, Cai YF,Hu FX. Digital gene expression analysis of reproductive toxicity of benzo [a] pyrene in male scallop chlamys farreri. Ecotoxicology and Environmental Safety 2014, 110,190-196.

Dudareva N, D'Auria JC, Nam KH, Natalia Dudareva, John C. D’Auria, Kyoung Hee Nam, Robert A. Raguso, Eran Pichersky. Acetyl-CoA: benzylalcohol acetyl-transferase an enzyme involved in floral scent production in Clarkia breweri. The Plant Journal $1998,14,297-304$. 
Defilippi, B.G., Dandekar, A.M., Kader, A.A. Relationship of ethylene biosynthesis to volatile production, related enzymes, and precursor availability in apple peel and flesh tissues.J. Agric. Food. Chem. 2005,53,3133-3141.

Elaine R. Mardis (2008) Next-Generation DNA sequencing methods. Annual Review of Genomics and Human Genetic.2008, 9,387-402.

Feussner, I.; Wasternack, C. The lipoxygenase pathway. Annual Review of Plant Biology,2002, 53,275-297.

Fabrizio Costa, Luca Cappellin, Elena Zini, Andrea Patocchi, Markus Kellerhals, Matteo Komjanc, Cesare Gessler, Franco Biasioli. QTL validation and stability for volatile organic compounds (VOCs) in apple. Plant Sci.2013,211:1-7.

Glenn TC. Field guide to next-generation DNA sequencers. Molecular Ecology Resource,2011, 11,759-769.

Gómez-Porras, J.L., Riano-Pachon, D.M., Dreyer, I., Mayer, J.E., Mueller-Roeber, B. Genome-wide analysis of ABA-responsive elements ABRE and CE3. BMC Genomics,2007,8,260-273.

Hossain MA, Huq E, Hodges TK. Sequence of a cDNA from Oryza sativa (L.) encoding the pyruvate decarboxylase 1 gene. Plant physio.1994,106,799.

Jennings, W., Sevenants, M. Volatile esters of bartlett pear:III. J. Food Sci. 1964, 29, 158163.

J. Speirs, E. Lee, K. Holt, K. Yong-Duk, N.S. Scott, B. Loveys, W. Schuch. Genetic manipulation of alcohol dehydrogenase levels in ripening tomato fruit affects the balance of some flavor aldehydes and alcohols. Plant Physio.1998,11,1047-1058.

Kou X, Liu X, Li J, Xiao H. Wang, J. Effects of ripening, 1-methylcyclopropene and ultrahigh-pressure pasteurisation on the change of volatiles in Chinese pear cultivars. J. Sci. Food Agric.2012, 92,177-183.

Li GP, Jia HJ, Li JH, Wang Q, Zhang MJ, Teng YW. Emission of volatile esters and transcription of ethylene and aroma-related genes during ripening of 'Pingxiangli' pear fruit (Pyrusussuriensis Maxim). Sci. Hortic.2014, 170,17-23. 
Lara I., Miro'R.M., T. Fuentes, G. Sayez, J. Graell, M.L. Lo'pez. Biosynthesis of volatile aroma compounds in pear fruit stored under long-term controlled-atmosphere conditions. POSTHARVEST BIOL. TEC.2003,29,29-39.

LEE KR, KIM SH, GO YS (2012) Molecular cloning and functional analysis of two FAD2 genes from American grape( Vitis labrusca L.). Gene 509(2):189-194.

Manriquez, D., El-Sharkawy, I., Flores, F.B., El-Yahyaoui, F., Regad, F., Bouzayen, M.,Latche, A., Pech, J.C (2006) Two highly divergent alcohol dehydrogenases of melon exhibit fruit ripening-specific expression and distinct biochemical characteristics. Plant Molecular Biology 61:675-685.

Matsui, M., Kurishita, S., Hisamitsu, A., Kajiwara, T. ( 2000) A lipid-hydrolysing activity involved in hexenal formation. Biochemical Society Transactions (28):857-860.

Michael Lay-Yee, Dean DellaPenna1,Gavin S. Ross. Changes in messenger-RNA and protein during ripening in apple fruit (Malus-domestica Borkh cv Golden Delicious). Plant Physio.1990,94,850-853.

Muna Ahmed Mohamed El Hadi, Feng-Jie Zhang, Fei-Fei Wu, Chun-Hua Zhou, Jun Tao. Advances in fruit aroma volatile research. Molecules, 2013,18,8200-8229

Marina Guillén, Maria Dolors Benaiges, Francisco Valero. Biosynthesis of ethyl butyrate by immobilized recombinant Rhizopus oryzae lipase expressed in Pichia pastoris. BIOCHEM ENG. J.2012, 65:1-9.

Ortiz A, Graell J, Lara I. Preharvest calcium sprays improve volatile emission at commercial harvest of 'Fuji Kiku-8' apples. Journal of J. Agric. Food. Chem. 2011,59,335-341.

Ortiz A, Echeverría G, Graell J, Lara I. The emission of flavour-contributing volatile esters by 'Golden Reinders' apples is improved after mid-term storage by postharvest calcium treatment. POSTHARVEST BIOL. TEC.2010, 57,114-123.

Qin GH, Tao ST , Cao YF , Wu JY, Zhang HP, Huang WJ ,Zhang SL. Evaluation of the volatile profile of 33 Pyrus ussuriensis cultivars by HS-SPME with GC-MS. Food Chem.2012, 134,2367-2382. 
Qin GH, Tao ST, Zhang HP, Huang WJ , Wu JY, Xu YL, Zhang SL. Evolution of the aroma volatiles of pear fruits supplemented with fatty acid metabolic precursors. Molecules2014, 19,20183-20196.

Qi XX, Wu J, Wang LF, Li LT, Cao YF, Tian LM, Dong XG, Zhang SL. Ientifying the candidate genes involved in the calyx abscission process of 'Kuerlexiangli' (Pyrus sinkiangensis $\mathrm{Yu}$ ) by digital transcript abundance measurements. BMC genomics $2013,14,727$.

Qin GZ, Wang YY, Cao BH, Wang WH, Tian SP. Unraveling the regulatory network of the MADS box transcription factor RIN in fruit ripening. The Plant J.2012,70, 243-255.

Rajesh K. Singh, Vidhu A. Sane, Aparna Misra, Sharique A. Ali, Pravendra Nath. Differential expression of the mango alcohol dehydrogenase gene family during ripening. Phytochemistry 2010,71,1485-1494.

Robert J. Schaffer, Ellen N. Friel, Edwige J.F. Souleyre, Karen Bolitho, Kate Thodey, Susan Ledger,Judith H. Bowen, Ma JH, Bhawana Nain, Daniel Cohen, Andrew P. Gleave, Ross N. Crowhurst,Bart J. Janssen, Yao JL, and Richard D. Newcomb. A genomics approach reveals that aroma production in apple is controlled by ethylene predominantly at the final step in each biosynthetic pathway. Plant Physio.2007,144,1899-1912.

Sandra Bensmihen, Jérôme Giraudat and Francois Parcy (2005) Characterization of three homologous basic leucine zipper transcription factors (bZIP) of the ABI5 family during Arabidopsis thaliana embryo maturation. J.Ex.B.2005,56,597-603.

Speirs, J., Correll, R., Cain, P. Relationship between ADH activity, ripeness and softness in six tomato cultivars. Sci. Hortic.2002, 93,137-142.

Song, J.; Bangerth, F. Fatty acids as precursors for aroma volatile biosynthesis in preclimacteric and climacteric apple fruit. POSTHARVEST BIOL. TEC. 2003,30,113121. 
Sanz C, Olias J M, Perez A G.Aroma biochemistry of fruits and vegetables[C] //PROCEEDINGS-PHYTOCHEMICAL SOCIETY OF EUROPE. Oxford University Press Inc., 1996,41,125-156.

S. Ramarethinam K. Latha and N. Rajalakshmi. Use of a fungal lipase for enhancement of aroma in black tea. FOOD SCI. TECHNOL. RES. 2002, 8,328-332.

Tesniere, C., Pradal, M., El-Kereamy, A., Torregrosa, L., Chatelet, P., Roustan, J.P.,Chervin, C. Involvement of ethylene signalling in a non-climacteric fruit: new elements regarding the regulation of ADH expression in grapevine. J.Ex.B.2004, 55,2235-2240.

Thompson, C.E., Salzano, F.M., Norberto de Souza, O., Freitas, L.B. Sequence and structural aspects of the functional diversification of plant alcohol dehydrogenases. Gene 2007,396,108-115.

Wang Li, Peter Tiffin, Matthew S. Olson. Timing for success: expression phenotype and local adaptation related to latitude in the boreal forest tree, Populus balsamifera. TREE GENET. GENOMES. 2014,10,911-922.

Wei SW, Zhang Y, Wang HW. Effect of organic fertilizer and bagging on fruit flavor quality of Yali pear. Plant Nutr. Fert. Sci. 2012,18,1269-1276. (in Chinese)

Wu J, Wang ZW, Shi ZB, Zhang S, Ming R, Zhu S, Khan MA, Tao ST, Korban SS, Wang H et al. The genome of pear (Pyrus bretschneideri Rehd.). Genome Res.2012, 23,396408.

Wyllie S.G., Fellman, J.K. Formation of volatile branched chain esters in bananas (Musa sapientum L.). Journal of J. Agric. Food. Chem. 2000,48,3493-3496.

Wójcik P., Skorupińska A., Filipczak J. Impacts of preharvest fall sprays of calcium chloride at high rates on quality and 'Conference' pear storability. Sci. Hortic.2014, 168,51-57.

Xi WP, Zhang B, Shen JY, Sun CD, Xu CJ, Chen KS (2012) Intermittent warming alleviated the loss of peach fruit aroma-related esters by regulation of AAT during cold storage. POSTHARVEST BIOL. TEC. 2012,74,42-48.

Zhang SL,Chen KS(2007) Molecular physiology of fruit quality development and regulation. Beijing: China Agriculture Press. (in Chinese) 
Zhang LP, SHen YX, Bu QZ and Ji SJ (2013) Effects of ripening, 1-methylcyclopropene on the metabolic pathways of aroma-related compounds in nanguo pear. J.Food Process. Pre.2013, 38, 1749-1758.

Zhang N, Zhang HJ ,Zhao B, Sun QQ , Cao YY, Li R, WuXX, Sarah Weeda, Li L, RenSX, Russel J. Reiter and GuoYD. The RNA-seq approach to discriminate gene expression profiles in response to melatonin on cucumber lateral root formation. J Pineal Res.2014, 56,39-50.

Zhang ZJ, Zhang Hw, Quan RD, Wang XC, Huang RF. Transcriptional regulation of the ethylene response factor LeERF2 in the expression of ethylene biosynthesis genes controls ethylene production in tomato and tobacco. Plant Physio.2009,150,365-377. 


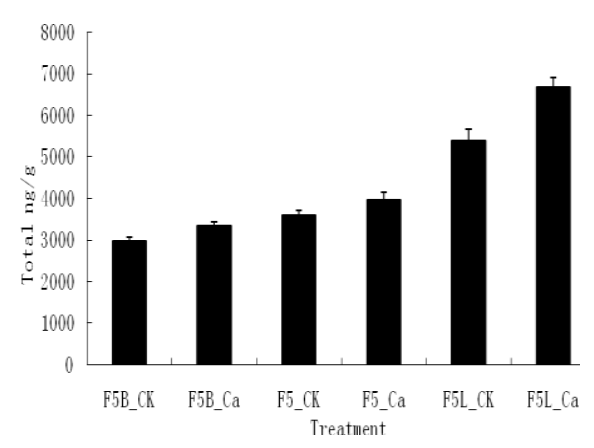

A

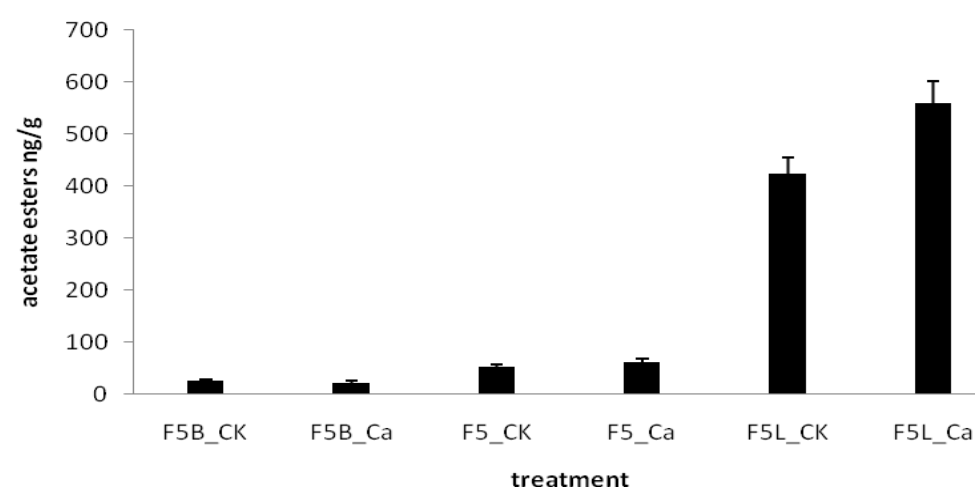

$\mathrm{C}$

B

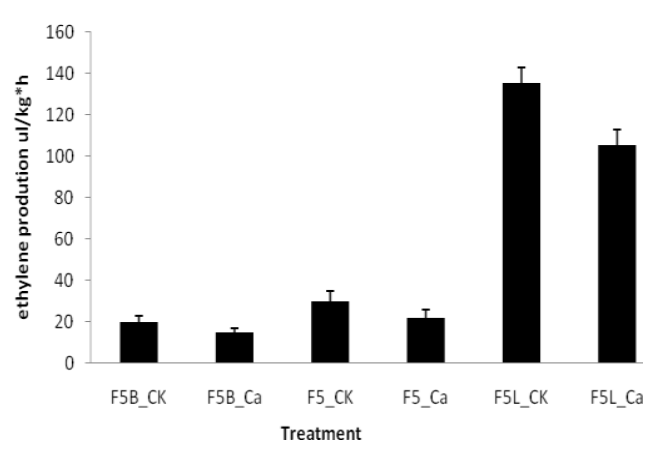

Fig. 1 A: Total volatile aroma compounds of 'Nanguoli' pear fruit. The total volatile aroma compounds of fruit increased significantly in post-harvest ripening, and calcium-treated fruit showed significant differences to the control. Results represent the mean of three replicates. B: Ethylene production of 'Nanguoli' fruit. C: Total quantity of acetate esters compounds in 'Nanguoli' pear fruit. 


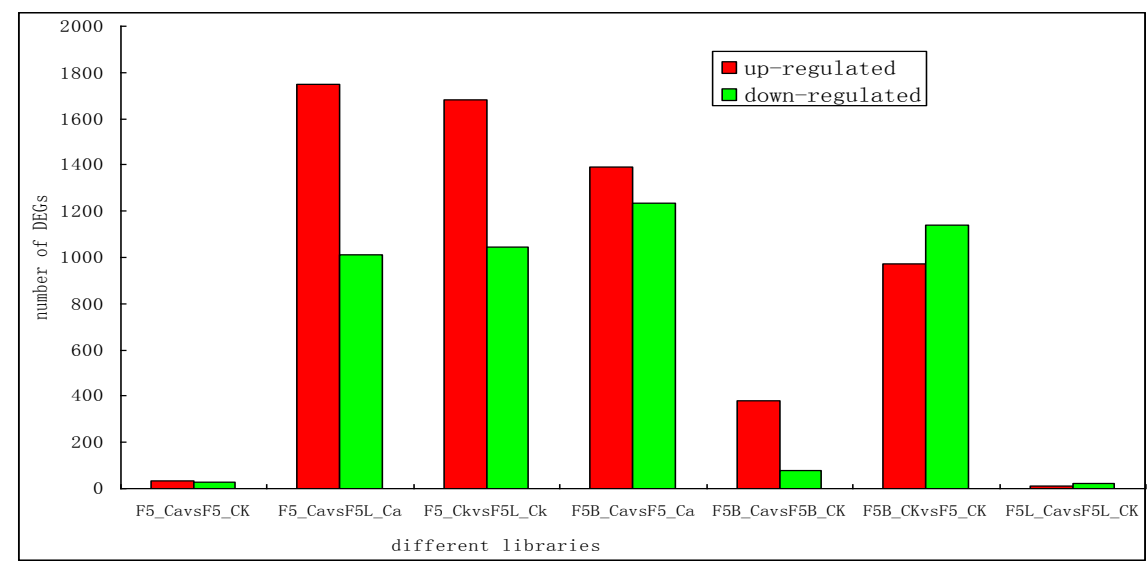

Fig. 2 Genes differentially expressed between different libraries. Up-regulated (red) and downregulated (green) genes were quantified. The results of seven comparisons are shown.
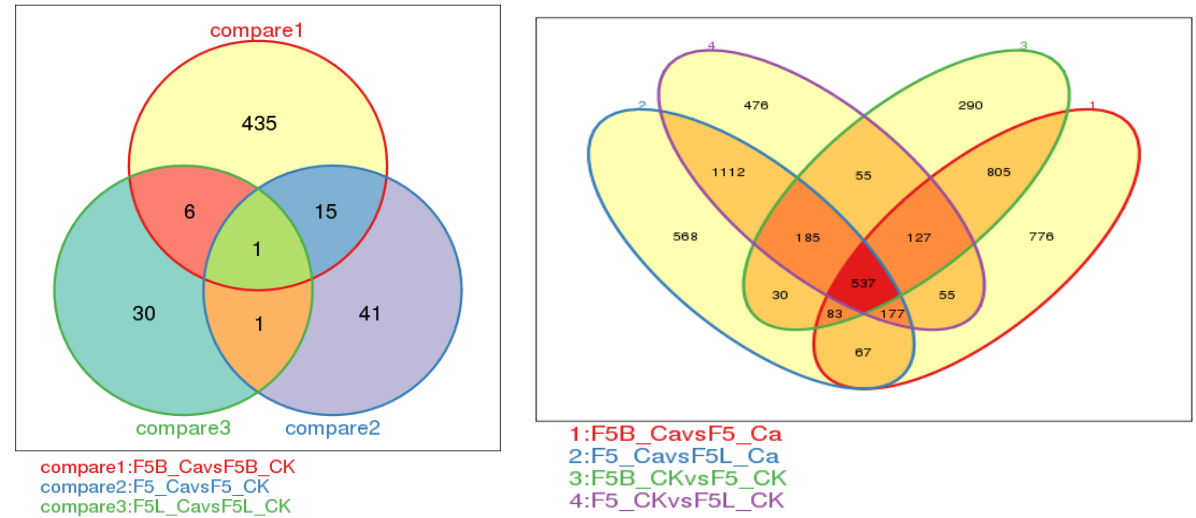

Fig. 3 Genes specifically expressed between different samples. Specifically expressed genes were quantified. The numbers of digital gene expression (DGE) genes of three comparisons between each two samples are shown 


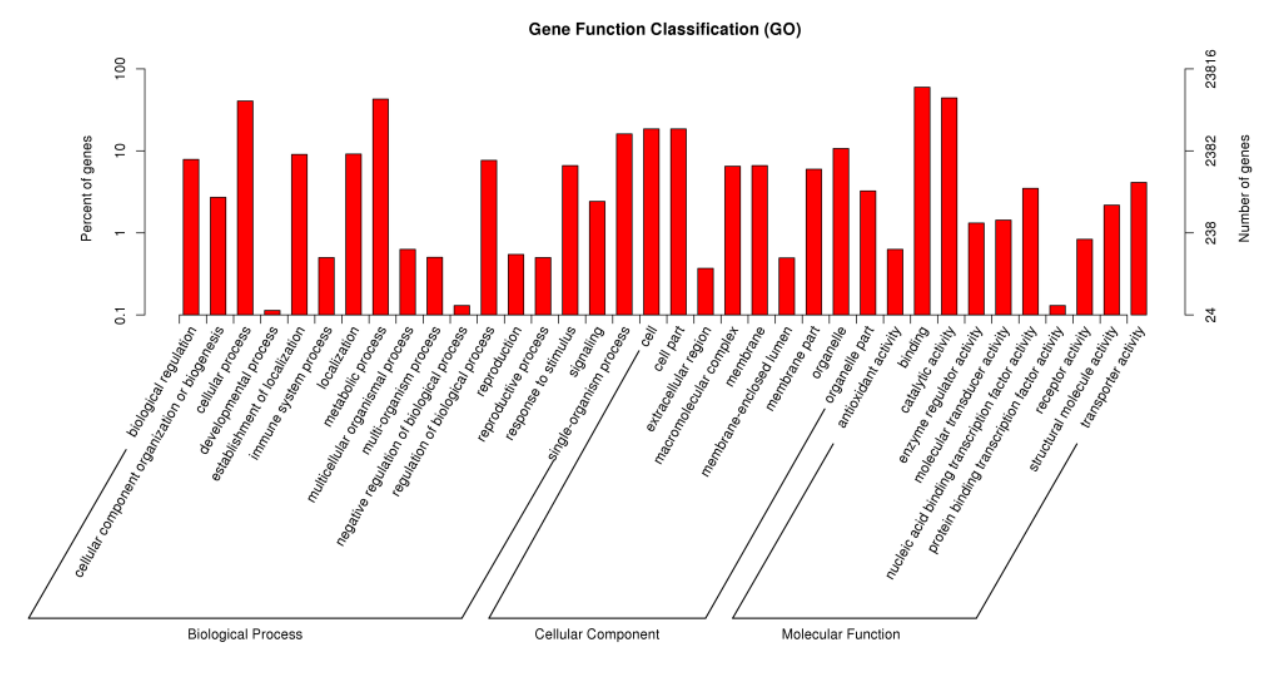

Fig. 4 Gene ontology categories of the genes identified. Y-axis (left) represents percentages of genes identified in this study; and $\mathrm{Y}$-axis (right) represent the actual gene number. The genes were annotated in three main categories: biological process, cellular component and molecular function (X-axis) 
Table 1. Description of 'Nanguoli' fruit samples used in this study

\begin{tabular}{ll}
\hline Sample code & \multicolumn{1}{c}{ Description } \\
\hline F5B_CK & Fruits without calcium treatment 5 days before commercial harvest \\
F5B_Ca & Fruits with calcium treatment 5 days before commercial harvest \\
F5_CK & Fruit without calcium treatment in commercial harvest \\
F5_Ca & Fruit with calcium treatment in commercial harvest \\
F5L_CK & Fruit (without calcium treatment) post-harvest ripening for 5 days in $20^{\circ} \mathrm{C}$ \\
F5L_Ca & Fruit (with Calcium treatment) post-harvest ripening for 5 days in $20^{\circ} \mathrm{C}$ \\
\hline
\end{tabular}

Table 2 Effects of calcium treatments on fruit quality of 'Nanguoli'

\begin{tabular}{lllllll}
\hline Aroma Volatiles & F5B_CK & F5B_Ca & F5_CK & F5_Ca & F5L_CK & F5L_Ca \\
\hline Firmness $\left(\mathrm{lb} / \mathrm{cm}^{2}\right)$ & $16.77 \pm 0.35$ & $17.23 \pm 0.17$ & $14.24 \pm 0.12$ & $15.01 \pm 0.32$ & $9.33 \pm 0.45$ & $12.89 \pm 0.37$ \\
Soluble solids content $(\%)$ & $9.1 \pm 0.12$ & $9.38 \pm 0.2$ & $11.4 \pm 0.17$ & $11.87 \pm 0.87$ & $11.79 \pm 0.31$ & $12.53 \pm 0.35$ \\
Titratable acids(\%) & $0.35 \pm 0.02$ & $0.37 \pm 0.01$ & $0.39 \pm 0.03$ & $0.41 \pm 0.04$ & $0.41 \pm 0.03$ & $0.44 \pm 0.04$ \\
\hline
\end{tabular}

Table 3 Effects of calcium treatments on aroma emission of 'Nanguoli' pear fruit (ng/g FW)

\begin{tabular}{|c|c|c|c|c|c|c|}
\hline Aroma Volatiles & F5B_CK & F5B_Ca & F5_CK & F5_Ca & F5L_CK & F5L_Ca \\
\hline \multicolumn{7}{|l|}{ Esters } \\
\hline Ethyl Acetate & $6 \pm 0.12$ & $6.6 \pm 0.2$ & $4.25 \pm 0.17$ & $19.1 \pm 2.87$ & $22.14 \pm 3.11$ & $68.34 \pm 4.35$ \\
\hline Propyl acetate & 0 & 0 & 0 & 0 & $1.62 \pm 0.17$ & 0 \\
\hline Butyl acetate & $163.05 \pm 7.33$ & $199.55 \pm 8.49$ & $268.95 \pm 9.55$ & $170.05 \pm 11.9$ & $70.44 \pm 5.48$ & $126.66 \pm 8.79$ \\
\hline Acetic acid, isobutyl ester & 0 & 0 & 0 & 0 & 0 & $2.82 \pm 0.17$ \\
\hline Pentyl acetate & $10.8 \pm 0.54$ & $9.6 \pm 0.78$ & $19.2 \pm 1.01$ & $14.35 \pm 1.11$ & $10.8 \pm 0.99$ & $12.96 \pm 1.07$ \\
\hline Hexyl acetate & $267.4 \pm 4.54$ & $257.2 \pm 5.78$ & $637.9 \pm 8.98$ & $589.9 \pm 19.9$ & $541.86 \pm 17.76$ & $388.2 \pm 11.37$ \\
\hline Heptyl acetate & 0 & 0 & 0 & 0 & $12.48 \pm 1.18$ & $8.28 \pm 0.66$ \\
\hline 2-Methyl-1-butyl acetate & 0 & 0 & $1.9 \pm 0.14$ & 0 & 0 & $4.26 \pm 0.17$ \\
\hline Caprylyl acetate & 0 & 0 & 0 & 0 & $6.72 \pm 0.55$ & $5.46 \pm 0.19$ \\
\hline Methyl pentanoate & 0 & 0 & 0 & 0 & 0 & $2.22 \pm 0.17$ \\
\hline Ethyl propanoate & 0 & 0 & 0 & 0 & $3.24 \pm 0.22$ & $38.46 \pm 2.15$ \\
\hline Butanoic acid, methyl ester & $3.2 \pm 0.21$ & $4 \pm 0.26$ & $2.3 \pm 0.15$ & $2.55 \pm 0.22$ & $8.7 \pm 0.69$ & $16.38 \pm 1.16$ \\
\hline Butanoic acid, ethyl ester & $4.5 \pm 0.23$ & $7.3 \pm 0.34$ & $81.15 \pm 3.33$ & $104.4 \pm 4.4$ & $184.68 \pm 7.77$ & $390.06 \pm 9.79$ \\
\hline Propyl butanoate & $5.2 \pm 0.43$ & $3.5 \pm 0.16$ & $9.25 \pm 0.74$ & $5.85 \pm 0.39$ & 0 & 0 \\
\hline Butanoic acid, butyl ester & $14.9 \pm 1.12$ & $12.05 \pm 1.17$ & $22.1 \pm 1.87$ & $18.95 \pm 2.23$ & 0 & $2.4 \pm 0.19$ \\
\hline Propyl butanoate & 0 & 0 & 0 & 0 & 0 & $1.86 \pm 0.1$ \\
\hline Hexyl butanoate & $257.5 \pm 11.78$ & $263.95 \pm 12.87$ & $319.3 \pm 13.37$ & $446.9 \pm 11.8$ & $20.4 \pm 1.17$ & $14.82 \pm 1.19$ \\
\hline Isobutyric acid, ethyl ester & 0 & 0 & 0 & 0 & $0.96 \pm 0.02$ & $3.9 \pm 0.1$ \\
\hline $\begin{array}{l}\text { Butanoic acid, 2-methyl-, } \\
\text { methyl ester, (.+/-.)- }\end{array}$ & 0 & 0 & 0 & 0 & $1.02 \pm 0.02$ & $2.64 \pm 0.17$ \\
\hline $\begin{array}{l}\text { Butanoic acid, 2-methyl-, } \\
\text { ethyl ester }\end{array}$ & 0 & 0 & 0 & 0 & $2.82 \pm 0.16$ & $27.6 \pm 1.11$ \\
\hline Hexyl 2-methylbutyrate & 0 & 0 & $3.65 \pm 0.44$ & $3.9 \pm 0.24$ & 0 & 0 \\
\hline Ethyl pentanoate & $1.5 \pm 0.09$ & $1.55 \pm 0.08$ & $2.8 \pm .23$ & $3.7 \pm 0.27$ & $14.64 \pm 1.332$ & $25.2 \pm 0.22$ \\
\hline Methyl caproate & $13.1 \pm 1.18$ & $11.55 \pm 1.15$ & $15.85 \pm 1.97$ & $14.5 \pm 1.19$ & $144.12 \pm$ & $191.94 \pm 3.3$ \\
\hline Ethyl caproate & $118.2 \pm 9.38$ & $93.15 \pm 8.67$ & $173.8 \pm 13.54$ & $186.6 \pm 14.43$ & $2295.72 \pm 25.66$ & $2755.08 \pm 29.86$ \\
\hline
\end{tabular}




\begin{tabular}{|c|c|c|c|c|c|c|}
\hline Propyl caproate & $2.6 \pm 0.15$ & $1.35 \pm 0.88$ & $8.7 \pm 0.89$ & $11 \pm 1.07$ & 0 & 0 \\
\hline Propyl hexanoate & 0 & 0 & 0 & 0 & $7.32 \pm 0.58$ & $7.74 \pm 0.67$ \\
\hline Hexyl hexanoate & $54.2 \pm 3.98$ & $69.85 \pm 3.76$ & $57.9 \pm 4.99$ & $128.45 \pm 9.95$ & $15.78 \pm 1.14$ & 0 \\
\hline Ethyl (E)-2-hexenoate & 0 & 0 & 0 & 0 & $4.86 \pm 0.38$ & $4.68 \pm 0.48$ \\
\hline Ethyl (E)-3-hexenote & 0 & 0 & 0 & 0 & $13.02 \pm 1.27$ & $17.16 \pm 2.1$ \\
\hline Ethyl enanthate & 0 & 0 & 0 & 0 & 0 & $8.58 \pm 0.79$ \\
\hline Methyl caprylate & 0 & 0 & 0 & 0 & $7.86 \pm 0.86$ & 0 \\
\hline Ethyl caprylate & $6.35 \pm 0.23$ & $5.45 \pm 0.26$ & 0 & 0 & $131.1 \pm 9.65$ & $55.02 \pm 1.99$ \\
\hline Ethyl (E)-2-octenoate & 0 & 0 & 0 & 0 & $12.72 \pm 1.19$ & $6.12 \pm 0.55$ \\
\hline 4-Octenoic acid, ethyl ether & 0 & 0 & 0 & 0 & $4.38 \pm 0.38$ & 0 \\
\hline $\begin{array}{l}\text { (E,Z)-Ethyl 2,4- } \\
\text { decadienoate }\end{array}$ & 0 & 0 & 0 & 0 & $14.92 \pm 1.18$ & $18.36 \pm 1.78$ \\
\hline \multicolumn{7}{|l|}{ Alcohols } \\
\hline Ethanol & 0 & 0 & $1.0 \pm 0.09$ & $2.8 \pm 0.25$ & $6.36 \pm 0.46$ & $12.84 \pm 1.09$ \\
\hline 1-Pentanol & 0 & 0 & 0 & $0.9 \pm 0.02$ & 0 & 0 \\
\hline 1-Hexanol & $29.75 \pm 1.11$ & $35.6 \pm 2.37$ & $37 \pm 1.7$ & $37.9 \pm 1.8$ & $9.42 \pm 1.11$ & $10.92 \pm 1.32$ \\
\hline (E)-2-Hexen-1-ol & 0 & 0 & 0 & $0.85 \pm 0.06$ & 0 & 0 \\
\hline 2-Butyl-1-octanol & 0 & 0 & $2.95 \pm 0.23$ & 0 & 0 & 0 \\
\hline \multicolumn{7}{|l|}{ Aldehydes } \\
\hline acetaldehyde & $178.01 \pm 3.39$ & $243.5 \pm 4.45$ & $200.7 \pm 12.43$ & $227.8 \pm 10.55$ & $115.31 \pm 6.76$ & $126.22 \pm 5.33$ \\
\hline Butanal,2-methyl- & $4.1 \pm 0.14$ & $2.65 \pm 0.12$ & 0 & 0 & $2.64 \pm 0.2$ & 0 \\
\hline Isobutyraldehyde & 0 & 0 & 0 & 0 & $9.54 \pm 0.89$ & 0 \\
\hline Pentanal & 0 & $0.9 \pm 0.04$ & 0 & $0.6 \pm 0.05$ & $2.28 \pm 0.11$ & 0 \\
\hline Hexanal & $500.04 \pm 7.45$ & $700.3 \pm 8.89$ & $606.2 \pm 24.11$ & $700.1 \pm 32.12$ & $1202.17 \pm 35.67$ & $1670.24 \pm 37.88$ \\
\hline (E)-2-Hexenal & $383 \pm 12.87$ & $408.4 \pm 14.44$ & $334 \pm 18.77$ & $365.05 \pm 15.3$ & $300.48 \pm 13.53$ & $355.53 \pm 15.56$ \\
\hline \multicolumn{7}{|l|}{ Ketones } \\
\hline 6-Methyl-5-heptene-2-one & $10.6 \pm 0.14$ & $7.6 \pm 0.26$ & $2.75 \pm 0.22$ & $3.45 \pm 0.25$ & $10.5 \pm 1.13$ & $7.02 \pm 0.56$ \\
\hline \multicolumn{7}{|l|}{ Alkanes } \\
\hline Hexane & $1.05 \pm 0.09$ & $2.2 \pm 0.21$ & 0 & $1.5 \pm 0.11$ & $3.06 \pm 0.32$ & $2.52 \pm 0.26$ \\
\hline 1,4-Dimethylbenzene & 0 & 0 & $3.6 \pm 0.18$ & $2.8 \pm 0.19$ & $1.8 \pm 0.09$ & $5.94 \pm 0.33$ \\
\hline alpha.-Farnesene & $875.85 \pm 19.5$ & $951.4 \pm 23.35$ & $388.5 \pm 11.76$ & $812.9 \pm 16.55$ & $159.78 \pm 9.35$ & $277.56 \pm 1.57$ \\
\hline Dodecane & $13.1 \pm 1.11$ & 0 & $20.5 \pm 1.67$ & $28.95 \pm 1.73$ & 0 & 0 \\
\hline Pentadecane & 0 & $5.1 \pm 0.24$ & 0 & $14.85 \pm 1.19$ & 0 & 0 \\
\hline Tetradecane & $12.75 \pm 1.06$ & $7.85 \pm 0.99$ & $21.25 \pm 1.68$ & $37.6 \pm 2.59$ & 0 & 0 \\
\hline $\begin{array}{l}\text { Tetradecane, } 2,6,10- \\
\text { trimethyl- }\end{array}$ & $4.85 \pm 0.34$ & 0 & $4.6 \pm 0.33$ & $1.5 \pm 0.14$ & 0 & 0 \\
\hline 3,6-Dimethyloctane & $4.55 \pm 0.48$ & $4.4 \pm 0.36$ & $7.65 \pm 0.57$ & 0 & 0 & 0 \\
\hline 4,6-Dimethyldodecane & $9.55 \pm 0.76$ & $6.6 \pm 0.57$ & $14.95 \pm 0.98$ & 0 & 0 & 0 \\
\hline
\end{tabular}

Note: 0 , not detected. Capitals denote a significant difference in a line for one cultivar at $\mathrm{P}<0.01$. Lowercase letters denote a significant difference in a line for one cultivar at $\mathrm{P}<0.05$, according to Duncan's test.

Table 4. Summary of the transcriptome reads for the normal control (CK) fruit and calciumtreated fruit. 


\begin{tabular}{lcclcccc}
\hline Sample name & Raw reads & Clean reads & clean bases & $\begin{array}{c}\text { Error rate } \\
(\%)\end{array}$ & Q20(\%) & Q30(\%) & $\begin{array}{c}\text { GC content } \\
(\%)\end{array}$ \\
\hline F5B_Ca & 16661134 & 16491915 & $1.65 \mathrm{G}$ & 0.03 & 97.31 & 91.77 & 46.44 \\
F5B_CK & 18800372 & 18582042 & $1.86 \mathrm{G}$ & 0.03 & 97.09 & 91.25 & 47.36 \\
F5_Ca & 18785368 & 18542126 & $1.85 \mathrm{G}$ & 0.04 & 97.03 & 91.13 & 47.57 \\
F5_CK & 17963096 & 17756965 & $1.78 \mathrm{G}$ & 0.03 & 97.1 & 91.26 & 46.95 \\
F5L_Ca & 19508289 & 19280515 & $1.93 \mathrm{G}$ & 0.03 & 97.11 & 91.29 & 47.24 \\
F5L_CK & 18166189 & 17928137 & $1.79 \mathrm{G}$ & 0.04 & 97.01 & 91.08 & 47.93 \\
\hline
\end{tabular}

Table 5. Reads with the reference genome comparison list

\begin{tabular}{lllllll}
\hline Sample name & F5B_CK & F5B_Ca & F5_CK & F5_Ca & F5L_CK & F5L_Ca \\
\hline Total reads & 18582042 & 16491915 & 17756965 & 18542126 & 17928137 & 19280515 \\
& 12974860 & 11461516 & 12244442 & 12772521 & 12715978 & 13649000 \\
Total mapped & $(69.82 \%)$ & $(69.5 \%)$ & $(68.96 \%)$ & $(68.88 \%)$ & $(70.93 \%)$ & $(70.79 \%)$ \\
& 1773623 & 1637743 & 1726271 & 1800542 & 1677989 & 1768196 \\
Multiple mapped & $(9.54 \%)$ & $(9.93 \%)$ & $(9.72 \%)$ & $(9.71 \%)$ & $(9.36 \%)$ & $(9.17 \%)$ \\
Uniquely & 11201237 & 9823773 & 10518171 & 10971979 & 11037989 & 11880804 \\
mapped & $(60.28 \%)$ & $(59.57 \%)$ & $(59.23 \%)$ & $(59.17 \%)$ & $(61.57 \%)$ & $(61.62 \%)$ \\
& 5599786 & 4905350 & 5249948 & 5482741 & 5498351 & 5920623 \\
Reads map to '+' & $(30.14 \%)$ & $(29.74 \%)$ & $(29.57 \%)$ & $(29.57 \%)$ & $(30.67 \%)$ & $(30.71 \%)$ \\
& 5601451 & 4918423 & 5268223 & 5489238 & 5539638 & 5960181 \\
Reads map to '-' & $(30.14 \%)$ & $(29.82 \%)$ & $(29.67 \%)$ & $(29.6 \%)$ & $(30.9 \%)$ & $(30.91 \%)$ \\
& 7670196 & 6819312 & 7352266 & 7669201 & 7689411 & 8222388 \\
Non-splice reads & $(41.28 \%)$ & $(41.35 \%)$ & $(41.4 \%)$ & $(41.36 \%)$ & $(42.89 \%)$ & $(42.65 \%)$ \\
& 3531041 & 3004461 & 3165905 & 3302778 & 3348578 & 3658416 \\
Splice reads & $(19 \%)$ & $(18.22 \%)$ & $(17.83 \%)$ & $(17.81 \%)$ & $(18.68 \%)$ & $(18.97 \%)$ \\
\hline
\end{tabular}

